\title{
CHAPTER 1.1
}

\section{THE EARTH'S ATMOSPHERE: AN INTRODUCTION}

\author{
Kleareti Tourpali ${ }^{1}$, Jean Lilensten ${ }^{2}$ and Roxana Bojariu ${ }^{3}$
}

\section{Introduction}

The Earth's atmosphere, the thin envelope of air that surrounds our planet, consists of a mixture of gases, extending from the surface of the Earth to the edge of space and tied to the Earth mainly by its gravitational force, and partly by the magnetic field at high altitude. It comprises mainly molecular nitrogen $(78 \%$ in mass) and molecular oxygen (21\%), with the other $1 \%$ composed of trace gases, named so because their concentrations are very small. Despite their small amount, carbon dioxide, ozone and water vapor are very important to the Earth's climate and can have a large impact on atmospheric processes. Above about $200 \mathrm{~km}$, atomic oxygen, although tenuous, becomes the major species while atomic hydrogen is the most abundant species above typically $1000 \mathrm{~km}$. Depending on the approach taken, there are various ways one can consider a distinction between atmospheric layers: composition, temperature, electromagnetic properties.

Although pressure and density decrease with altitude in the atmosphere, temperature remains relatively constant or even increases with altitude at certain regions, so it may well be used to distinguish between atmospheric layers. Based on temperature, the atmosphere is divided into four layers: the troposphere, stratosphere, mesosphere and thermosphere, as illustrated in Figure 1. Based on composition and mixing, the atmosphere can be divided into two major layers, the homosphere and heterosphere, defined by whether the atmospheric gases are well mixed. The homosphere, starting from surface, includes the troposphere, stratosphere, mesosphere and the lowest part of the thermosphere. From there on lies

\footnotetext{
1 Lab of Atmospheric Physics, Aristotle University, Campus Box 149, Thessaloniki 54124, Greece

${ }^{2}$ Institut de Planétologie et d'Astrophysique de Grenoble (IPAG), Bâtiment D de physique, BP 53, 38041 Grenoble cedex 9, France

3 National Meteorological Administration, Sos. Bucuresti-Ploiesti 97, 013686 Bucureşti, Romania
} 
the heterosphere where the chemical composition varies with altitude, and gases are stratified due to their molecular weight. In this section, we discuss the properties of the atmospheric layers distinguished by both composition and temperature profiles.

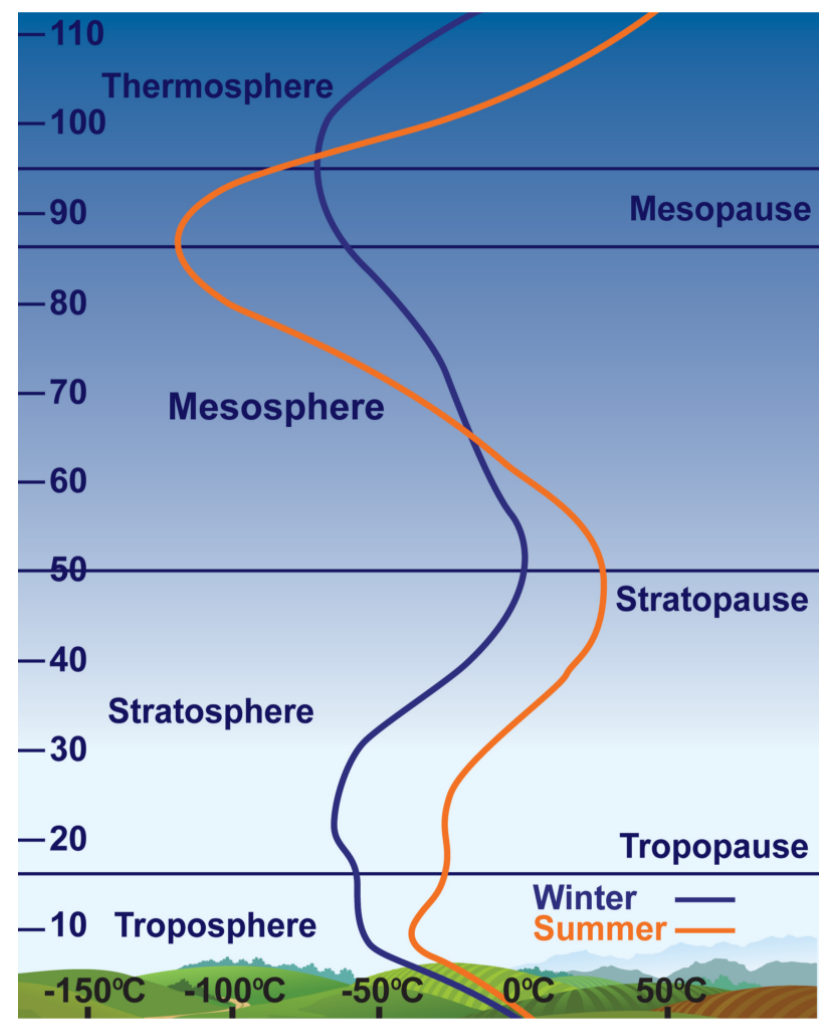

Fig. 1. Structure of the atmosphere and its layers based on temperature distribution during summer (orange line) and winter (blue line). (Adapted from http://www. athena-spu.gr/).

\section{The homosphere}

The amount of solar radiative energy that arrives perpendicularly to the Earth is on average $1361 \mathrm{~W} \mathrm{~m}^{-2}$; this quantity is called Total Solar Irradiance (TSI). However, taking into account the spherical shape of the Earth, this value must be divided by 4 (i.e., $340 \mathrm{~W} \mathrm{~m}^{-2}$ ) to obtain the overall mean amount of energy that reaches the Earth.

About $50 \%$ of this energy is used to heat the surface, $20 \%$ is absorbed in the atmosphere and $30 \%$ is reflected back to space (the albedo is the ratio between the amount of energy reflected or diffused and the amount received. It approaches 1 for snow, for instance, and therefore amounts to 0.3 on average on the surface of the Earth). 
The energy absorbed on the surface of the Earth heats the ground and then, by conduction and convection, the air in the lowest layer of the atmosphere. It also leads to considerable evaporation from the oceans and lakes. This phenomenon, which is amplified by the transpiration of plants, results in a rate of relative humidity of more than $50 \%$ on average on the globe in the troposphere, which contains $99 \%$ of atmospheric water vapor.

\subsection{The troposphere}

The term troposphere is a Greek-derived term (tropein - to change, circulate or mix), indicating the turbulent nature of this layer where weather systems circulate air masses and move around almost all content of atmospheric water vapor. Water vapor concentration varies from trace amounts in polar regions to nearly $4 \%$ in the tropics. Most clouds are present in this layer, too. The troposphere contains around $75-80 \%$ of the total mass of the Earth's atmosphere. As mentioned above, the main constituents of the atmosphere are nitrogen, oxygen, argon and traces of ozone, and other gases and particles. Carbon dioxide is also present in small amounts, but its concentration has increased by more than $40 \%$ in comparison with the pre-industrial level (e.g., 1750) due to human activities. Like water vapor, carbon dioxide is a greenhouse gas, which traps a part of the Earth's heat in the troposphere.

The troposphere extends upward to about 16-20 km above the Equator, and to about $7-8 \mathrm{~km}$ above the poles. The tropospheric thickness depends on latitude, season, and diurnal cycle. It is highest in summer and lowest in winter. This seasonal effect is strongest at mid-latitudes.

The troposphere is mainly heated from the Earth's surface warmed by solar radiation. The uneven heating of the Earth surface by the Sun (which warms land and ocean at the Equator more than at the poles) leads to large-scale patterns of winds that move heat and moisture around the globe. Atmospheric circulation is deflected by the Earth's rotation as it goes between the poles and Equator, creating weather systems and belts of surface winds. The warmed air tends to rise leading to convection which is the mechanism responsible for the vertical transport of heat in the troposphere while horizontal heat transfer is due to advection. Air convection generates clouds and ultimately precipitation when liquid or solid water particles grow large enough in size to fall toward the surface. Temperature, pressure, air density and water vapor content in the troposphere decrease with height. Air temperature decreases with height at a mean lapse rate of about $-6.5 \mathrm{C} \mathrm{km}^{-1}$, up to the tropopause (the boundary between the troposphere and stratosphere). However, sometimes temperature can increase with height within limited tropospheric layers leading to temperature inversions, which prevent the vertical mixing of air. Such atmospheric stability can lead to air pollution episodes at ground level. The temperature is constant in the tropopause. In the troposphere, winds increase with height leading to jet streams which usually meander in the upper parts, moving air at speeds of about $100 \mathrm{~km} \mathrm{~h}^{-1}$, just below the tropopause. Atmospheric circulation in the 
upper troposphere strongly influences weather systems in the lower troposphere, too.

The troposphere influences the stratosphere - the next layer beyond tropopause - through atmospheric waves that propagate upward. A number of studies show that this wave forcing leads to slowly evolving changes in stratospheric circulation, affecting polar areas (polar vortex; see next paragraph), which in turn can feed back to tropospheric phenomena (such as the Arctic/North Atlantic Oscillation) that shape anomalous weather regimes in winter.

\subsection{The stratosphere}

The stratosphere is a region where the air temperature increases gradually to the stratopause (found at an average height of $50 \mathrm{~km}$ ), the area marking the border to the mesosphere (Figure 2). Due to the increase of temperature with height through the whole stratosphere, warmer air lies above colder air. Hence, convection is not favored. The atmospheric conditions are stable and vertical mixing is suppressed. Only small amounts of water vapor enter the stratosphere (due to their freezedrying at the tropopause), so the stratosphere is a very dry region.

Ozone plays the major role in regulating the thermal regime of the stratosphere. The stratospheric region with the highest ozone concentration is known as the "ozone layer" or "ozonosphere", located at heights roughly between 15 and $35 \mathrm{~km}$ extending over the entire globe, with some variation in altitude and thickness. Its peak concentration occurs at heights from 26 to $28 \mathrm{~km}$ (or slightly higher) in the tropics and from about 12 to $20 \mathrm{~km}$ toward the poles. Typical ozone concentrations at its peak are about 10 parts per million by volume (ppmv), reaching to higher values over the tropics. Temperature increases with ozone concentration, as solar energy is converted to kinetic energy when ozone molecules absorb ultraviolet radiation, resulting in heating of the stratosphere. Temperature increases also due to thermal energy released during the ozone formation cycle. The reaction of oxygen $(\mathrm{O})$ and oxygen molecules $\left(\mathrm{O}_{2}\right)$ that produces ozone is exothermal; heat is released and the stratosphere warms. As ozone is the main gas involved in radiative heating of the stratosphere, its solar-induced variations can directly affect the radiative balance of the stratosphere with indirect effects on circulation. A detailed account of solar-induced variations in the stratosphere and the ozone layer is given in Chapter 4.1.

Intense interactions take place among radiative, dynamical and chemical processes, in which the horizontal mixing of gaseous components proceeds much more rapidly than in vertical mixing. The horizontal structure of the stratosphere and the stratospheric circulation present a number of interesting features. Air rises in the tropical region from the troposphere into the stratosphere, is transported upward and poleward in the stratosphere, and descends in stratospheric middle and polar latitudes. This circulation, called the Brewer-Dobson Circulation, is strong in the respective winter hemisphere and transfers ozone and other trace constituents from the tropics to the polar regions, as illustrated in Figure 2. The polar vortex is a large-scale circumpolar cyclonic circulation (strong west-to-east 
winds that circle the polar regions). The polar vortex extends from the upper levels of the troposphere through the stratosphere and into the mesosphere (above $50 \mathrm{~km}$ ) and is associated with low temperature and ozone values. Polar vortices occur over both hemispheres, strongest during the respective winter season, when the pole-to-equator temperature gradient is strongest. They play an important role in the coupling between the troposphere and stratosphere (e.g., Arctic Oscillation) and in ozone depletion (e.g., Antarctic Ozone Hole).

Other important features of the stratosphere are the quasi-biennial oscillation (QBO) and the Sudden Stratospheric Warmings (SSWs), both of which affect circulation and transport of tracers, such as ozone and water vapor (see the Glossary for their brief descriptions).

\subsection{The mesosphere}

The mesosphere's (middle sphere) average location is from about 50 to $90 \mathrm{~km}$ above the Earth's surface, and is characterised by temperature decreasing with height. Thus, the mesosphere is warmer at its lowest level (just above the stratopause), and becomes coldest at its highest level. The upper limit of the mesosphere, the mesopause, is at the altitude at which the temperature reaches a minimum. The mesopause, a region that experiences large height variations, is located at altitudes between 85 and $100 \mathrm{~km}$, depending on latitude and season. The average mesopause temperature is about $-90^{\circ} \mathrm{C}$ (Figure 1 ), but the lowest temperatures

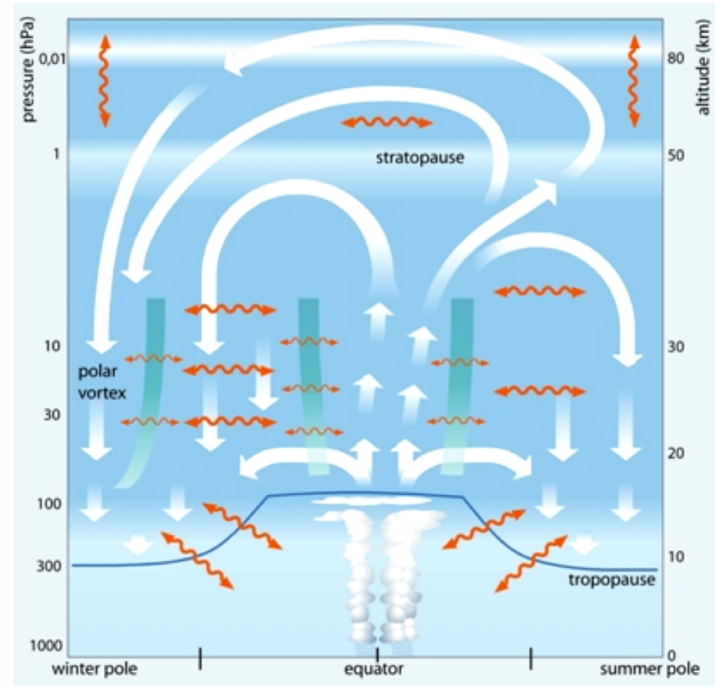

Fig. 2. Schematic representation of the Brewer-Dobson Circulation and Middle Atmosphere Transport. Arrows show the different pathways of air mass transport in the stratosphere and the stratosphere-troposphere exchange. Retrieved from Brewer-Dobson Circulation, (2011) http://www.eoearth.org/view/article/150779/. 
are found over the poles during the summer season, where the temperature may often drop below $-100{ }^{\circ} \mathrm{C}$ (at the same time, the other (winter) pole may have higher temperatures by several ${ }^{\circ} \mathrm{C}$ ), so the summer polar mesopause is the coldest level in the whole atmosphere.

The percentage of oxygen, nitrogen, and carbon dioxide in the air in the mesosphere is almost the same as that in the lower atmospheric levels, but the density of air is much less. The water vapor content is very small, and the mesosphere contains higher percentages of ozone than the lower levels. The mesosphere is the coldest among the atmospheric layers, so that the small amounts of water vapor that are present there are frozen into ice crystal clouds. These are the highest clouds in the Earth's atmosphere, called Noctilucent Clouds and can be observed after sunset at high latitudes during summer.

The mesosphere is a region where solar radiation and energetic particles contribute to downward energy transfer, and gravity and planetary waves along with the atmospheric tides transfer energy upwards from the stratosphere. Strong variations occur in the mesosphere as a function of height, both in the physical processes as well as in the chemical composition, especially in the vicinity of the mesopause.

The mesosphere together with the lower thermosphere (sometimes referred to as Mesosphere-Lower Thermosphere or MLT) is the region where vertical turbulent mixing is gradually replaced by molecular diffusion as we move to higher altitudes. The amount of solar radiation available to photolyse atmospheric molecules increases rapidly; molecules dominate the atmospheric composition at lower levels and atomic species, which are lighter, are abundant in the upper layers.

The major contributions to the energy budget are the absorption of solar radiation by ozone and molecular oxygen, the emission of infrared radiation by $\mathrm{CO}_{2}$, dissipative processes from the interaction with gravity waves, and transport of heat through advection. The mesosphere is also the layer in which many meteors burn up when they enter the Earth's atmosphere, as a result of the collision with some of the gas molecules present in this layer.

The stratosphere and mesosphere together are in many cases referred to as the "middle atmosphere". In all the "low" layers we have just described, molecules and atoms mingle, producing a homogeneous gas. The concentration is roughly $10^{25}$ particles per cubic meter at ground level and $10^{19}$ particles per cubic meter at the homopause (or turbopause), which is its upper limit.

\section{The heterosphere, the thermosphere, the ionosphere}

The heterosphere begins above the homosphere. It only became possible to explore the properties of this part of the atmosphere with the advent of radio communications in the twentieth century. Subsequently, sophisticated radar techniques and measurements by satellite revealed a complex, dynamic medium, a gas consisting of a mixture of electrically charged particles and neutral particles. This sheath still raises many questions about the part it plays in the ecosystem of the Earth and in the emergence of life on Earth. 
In the heterosphere, the concentration of molecules and atoms becomes very low and each component behaves as if it were alone. Here, the perfect gas behaviour of the whole of the homosphere now applies separately to nitrogen, oxygen, and hydrogen, with a fundamental difference: each has its own scale height. The immediate result is a variation in their exponential concentration but with different decrease rates. At about $80 \mathrm{~km}$, molecular nitrogen is predominant, followed by molecular oxygen. Above about $250 \mathrm{~km}$, atomic oxygen prevails. However, at around 1,000 km, hydrogen becomes the most abundant element: since there is no longer a convection mixture, the heaviest elements stay in the lower layers and the lighter elements "float" above them.

One fundamental feature of the heterosphere is that it constitutes a filter for radiation in the extreme ultraviolet (EUV). These emissions, strongly correlated with solar activity, are absorbed in ionisation, excitation, dissociation and heating. We are therefore in a medium that is very different from the matter with which we are familiar, a mixture of neutral gas, ions that are more or less energised and electrons. The neutral gas has been given the name of thermosphere. The combination of ionised gas, ions and electrons is the ionosphere. The mixture of the ionosphere and thermosphere is a plasma called the "upper atmosphere". Its properties are quite different from those of a classic gas consisting of neutral particles, since the movement of the charged particles is sensitive to the electrical and magnetic fields. However, the proportion of charged particles remains low in comparison with that of the neutral particles: about one billionth at an altitude of $100 \mathrm{~km}$ and one tenth at around $1,000 \mathrm{~km}$.

The heating of the upper atmosphere is indirect: the temperature of the atmosphere is increased by the friction of energised particles against those that have not been energised and by chemical reactions, not by direct interaction between the atmosphere and solar radiation. The temperature rises considerably above the mesosphere, roughly 8 to $10^{\circ} \mathrm{C}$ per kilometer between 100 and $150 \mathrm{~km}$. This is a positive temperature gradient: hot air no longer rises since it is already on top. Convection is inhibited and only conduction can transfer energy from one layer to the next.

This heating is effective as far as $200-300 \mathrm{~km} \mathrm{up}$; above this altitude, the atmosphere is too thin to conduct heat. Higher up, the temperature becomes constant and is then called exospheric temperature $T_{\infty}$. Its typical value is between 1,000 and 1,200 $\mathrm{K}$ during a quiet period but it can exceed 2,000 $\mathrm{K}$ during periods of high solar activity ${ }^{4}$. This explains why this part of the atmosphere is called the thermosphere.

When the Sun is quiet, the temperature is about $1,000 \mathrm{~K}$ for the ions at $400 \mathrm{~km}$ and 1,500 $\mathrm{K}$ for electrons. However, these are low values compared with those that occur when the Sun is particularly active: the temperature of the ions can then be as high as $2,000 \mathrm{~K}$ and that of the electrons $3,000 \mathrm{~K}$ at the same altitude. The four effects of solar radiation on the high atmosphere (ionisation,

\footnotetext{
4 Temperatures in the lower atmosphere are usually expressed in units of ${ }^{\circ} \mathrm{C}$, whereas higher
} up, Kelvin $(\mathrm{K})$ are used. Both temperatures are related by $T\left[{ }^{\circ} \mathrm{C}\right]=T[\mathrm{~K}]-273.15$. 
dissociation, excitation, heating) depend on the intensity of the radiation and therefore on solar activity.

\section{The magnetosphere}

Above an altitude of about $600 \mathrm{~km}$, and up to several terrestrial radii, particle concentration becomes so low that their behaviour is no longer a function of collisions but is a consequence of the configuration of the magnetic field. The nature of the environment changes and becomes the magnetosphere. The magnetosphere is not, strictly speaking, a part of the atmosphere, but rather part of the Earth space environment. However, as this book is dealing with the impact of the solar variability on the climate, the magnetosphere cannot be ignored.

The solar wind and the geomagnetic field interact in various ways. One is described in terms of both magnetic pressure (of the Earth's field) and kinetic pressure (of the solar wind). Furthermore, the magnetic field influences the charged particles of the solar wind, creating a large series of currents inside of the magnetosphere. We cannot describe all of them, and will only mention some that are relevant for this book.

The front of the magnetopause is located at approximately 10 terrestrial radii from the surface of our planet (the Earth's average radius is $6371 \mathrm{~km}$ ). This distance increases to approximately 13 terrestrial radii during periods of low solar activity, and may occasionally drop to 6 radii during periods of high activity, i.e., when massive perturbations called "coronal mass ejections" are ejected from the Sun. On its flanks, the magnetopause is at about 15 terrestrial radii from the Earth. On the night side, it is shaped like the tail of a comet. On the outside, the space is subjected to the solar wind and the interplanetary magnetic field. Inside the magnetosphere, the terrestrial magnetic field is the controlling force. The magnetopause stands like a porous barrier between the two. On the night side, the magnetopause closes at several dozen of terrestrial radii.

A small fraction only of the solar wind can penetrate the magnetosphere. When its charged particles come closer to the Earth, they encounter an increasingly intense magnetic field. They end up being stopped, typically at a distance ranging from two to ten terrestrial radii, thereby creating a ring of current, also called Van Allen Belt, which surrounds the Earth at low latitudes. This ring is located between four and seven terrestrial radii; its current density is typically $10^{8} \mathrm{~A} \mathrm{~m}^{-2}$, and $90 \%$ of its particles have energies ranging from $10 \mathrm{keV}$ to $250 \mathrm{keV}$, which is about 4 orders of magnitude more than in the solar wind. The influence of the ring current on Earth is noticeable at low latitudes, in equatorial regions. These particles are finally trapped and follow the magnetic field lines toward the south or the north magnetic poles. The rings plotted by the feet of the belt are located at high latitudes, between $65^{\circ}$ and $75^{\circ}$ North or South. They are called the auroral ovals, and coincide with the regions where auroras are most frequently observed.

Below five to six terrestrial radii from the surface of the Earth, the atmosphere is carried along by the rotation of the planet; this is known as "corotation". This 
area is called the plasmasphere. Here the density varies between 10 billion particles per cubic meter $\left(10^{10} \mathrm{~m}^{-3}\right)$ at the top of the ionosphere, at approximately 1,000 $\mathrm{km}$, and 100 billion $\left(10^{8} \mathrm{~m}^{-3}\right)$ at its outer boundary, the plasmapause.

Inside the plasmasphere there is another radiation belt, the first Van Allen belt, or inner belt. It is in a compact region centered above the equator, at an altitude of a few thousand kilometers. The origin of the belt is not to be found in the solar wind but primarily in the ionisation triggered by cosmic rays.

\section{Conclusion}

In the description of the Earth's atmosphere presented in this chapter, we have tried to give the reader a brief insight of the basic properties, composition, chemistry and processes that govern the atmospheric layers. This may seem as describing an onion: a series of layers that do not communicate with each other. However, this is not the case, as, and in order to understand the impact of solar activity on climate on its full extent, one should keep in mind that the Earth and its atmosphere constitute an integrated complex system of interlinked and interacting physical, chemical and biological processes. Our introduction here may serve as the background information for the understanding of the following chapters. Many processes occur at the interfacing boundaries between layers, and the physics of the system lies in the interactions. Moreover, this description is static, while the atmosphere is dynamic: it is constantly changing and interacting in a multitude of ways that connect layers to one another.

\section{Further reading}

Andrews, D. G., Holton, J. R., and Leovy, C. B.: 1987, Middle Atmosphere Dynamics, New York, Academic Press.

Brasseur, G. P. and Solomon, S.: 2005, Aeronomy of the middle atmosphere: Chemistry and Physics of the Stratosphere and Mesosphere, 3rd edition, Berlin, Springer.

Danielson, E. W., Levin, J. and Abrams E., 2003: Meteorology, New York, McGrawHill.

Holton, J. R., Curry, J. A., and Pyle, J. A. (editors): 2002, Encyclopedia Of Atmospheric Sciences, Ney York, Academic Press.

Lilensten, J., and Bornarel, J., 2006: Space weather, environment and societies, Berlin, Springer.

Peixoto, J. P., and Oort, A. H., 1992: Physics of Climate, New York, American Institute of Physics.

Wallace J. M. and P V. Hobbs, 2006: Atmospheric Science: An Introductory Survey, New York, Academic Press. 
